\title{
Omuz cerrahisinde tarihsel gelişim
}

\section{Historical evolution of the shoulder surgery}

\author{
Ertuğrul Akşahin ${ }^{1}$, İsmail Karasoy ${ }^{2}$ \\ ${ }^{1}$ Ortopedi Ankara Kliniği, Ankara \\ ${ }^{2}$ Gümüşhane Devlet Hastanesi, Ortopedi ve Travmatoloji Kliniği, Gümüşhane
}

\begin{abstract}
Omuz cerrahisi günümüz ortopedi ve travmatoloji rutininin önemli bir parçasını oluşturmaktadır. Açık ya da kapalı (artroskopik) instabilite ve rotator kılıf cerrahisi, omuz cerrahisinde en sık kullanılan tekniklerdir. Omuz yaralanmaları (omuz çıkıkları) ve bu yaralanmalara müdahaleler hakkındaki ilk kayıtlara M.Ö. 3000 yıllarında antik Mısır hiyerogliflerinde rastlanmaktadır. Omuz cerrahisinin tarihsel süreci incelendiğinde instabilite cerrahisinin rotator kılıf cerrahisine göre daha önce yapıldığı ve geliştirildiği görülmektedir. Bu süreçler 19. yy sonlarında anatomik çalışmalarla başlayıp 20. yy ilk yarısında açık tekniklerle cerrahi uygulamalarıyla devam etmektedir. Özellikle 1970'lerde Watanabe ve ark.'nın diz artroskopisi üzerine yaptıkları çalışmaları omuz cerrahisi evrimini de etkileşmiş ve zamanla artroskopik omuz cerrahisi açık cerrahilere göre daha fazla kullanılan bir yöntem olmaya başlamıştır.
\end{abstract}

Anahtar sözcükler: omuz cerrahisi; omuz instabilitesi; rotator kılı; artroskopi

\begin{abstract}
Shoulder surgery is an important part of routine orthopedics and traumatology practice. Open or closed (arthroscopic) instability and rotator cuff surgery are the most commonly used surgical techniques in shoulder surgery. The very first record of shoulder injuries (dislocation) and their treatment were found in ancient Egypt hieroglyphs at 3000 B.C. It is clearly seen that instability surgery was done and developed prior to rotator cuff surgery when the history of shoulder surgery has been analyzed. These processes began with anatomical studies in late 19th century and continued with open surgery procedures in early 20th century. In the 1970s, especially studies by Watanebe et al. on knee arthroscopy affected shoulder surgery evolution too and by time arthroscopic surgery became a more commonly used methods than open procedures in shoulder surgery.

Key words: shoulder surgery; shoulder instability; rotator cuff; arthroscopy
\end{abstract}

Omuz yaralanmalarına cerrahi yaklaşım tüm diğer ortopedi ve travmatoloji alanlarında olduğu gibi 19. yy sonlarında anatomik çalışmalarla başlayıp, 20. yy'nin ilk yarısında açık teknik tedavi uygulamaları ile devam etmiştir. Yirminci yüzyılın ikinci yarısından sonra da, gelişen teknik altyapı ve implant teknolojisi sayesinde artroskopik ve prostetik (artroplasti) cerrahi tedavi uygulamaları çok daha üst bir seviyeye taşınmıştır. ${ }^{[1,2]}$

Bu yazıda instabilite ve rotator kılıf patolojilerinin cerrahi tedavi süreçlerinin tarihsel gelişimi ve değişimi incelenecektir.

\section{OMUZ ARTROSKOPISI ÖNCESI DÖNEM}

On dokuzuncu yüzyılın başında ilk omuz cerrahisi uygulamalarını, tekrarlayan omuz çıkıklarının tedavi-

İletişim / Contact: Prof. Dr. Ertuğrul Akşahin •E-posta / E-mail: ertugrul_aksahin@hotmail.com

ORCID iD: Ertuğrul Akşahin, 0000-0001-5771-8476 • İsmail Karasoy, 0000-0001-9487-3774

Geliş / Received: 31 Ocak 2022 • Kabul / Accepted: 15 Şubat 2022 


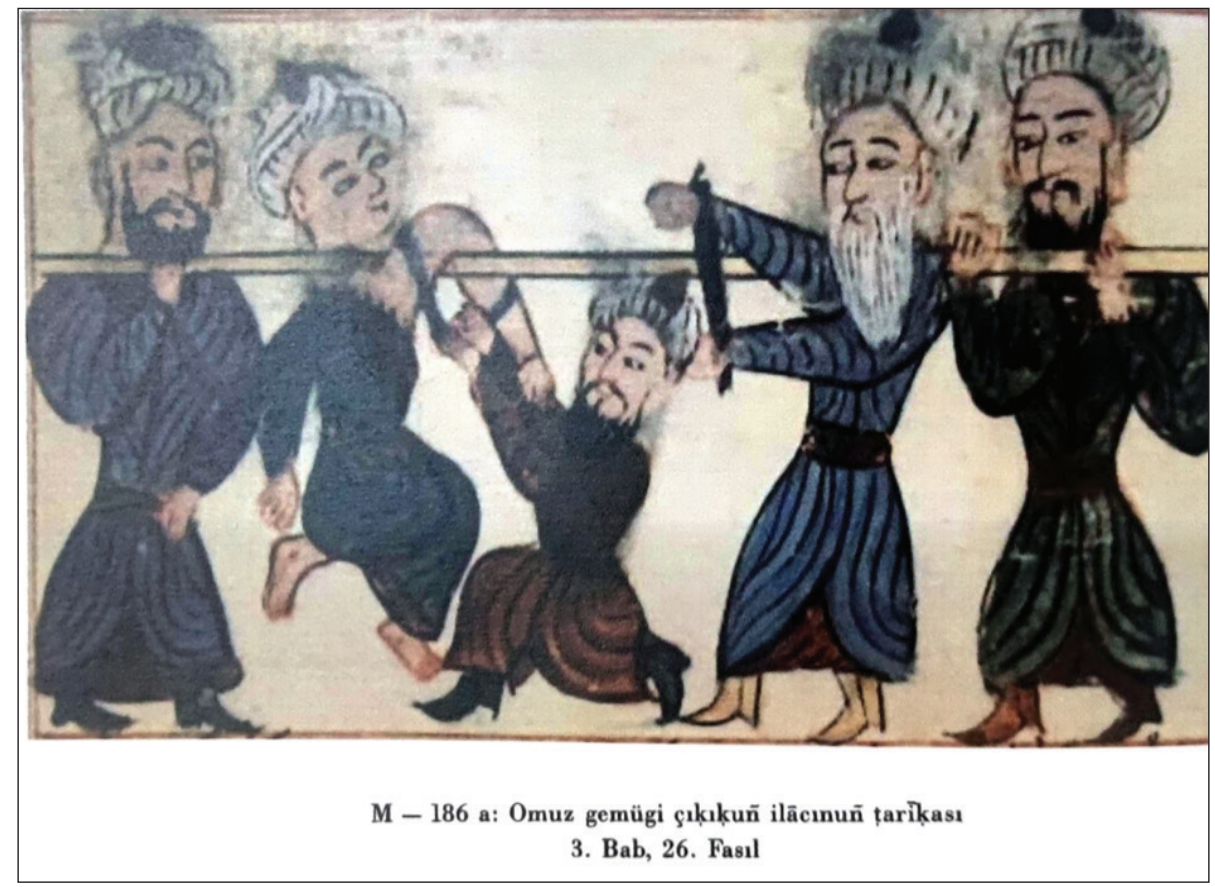

Şekil 1. Şerafettin Sabuncuoğlu'nun kitabında gösterilen omuz çıkığı redüksiyon tekniği (Türk Tarih Kurumu'nun izni dâhilinde kullanılmıștır).[4]

sinde görmekteyiz. Karl August Weinhold, 1819 yılında rekürren omuz çıkıklarındaki ilk cerrahi müdahaleyi yapmıştır. Weinhold bu işlemde fibröz bantları subkutanöz yaklaşımla kesiyordu. Weinhold'un bu tekniğinden sonra başka yöntemler tanımlanmış olsa da o dönemde tekrarlayan çıkıklar genellikle ya Alman cerrah Cramer'ın tanımladığı humerus başı rezeksiyonu ya da Çek cerrah Eduard Albert'in tanımladığı skapulohumeral artrodez ile palyatif tedavi edilmekteydi. ${ }^{[2]}$ Fransız cerrahlar Broca ve Hartman 1890 yılında yayımladıkları anatomi çalışmalarında omuz instabilitesi konusunda devrim yarattılar. Bu çalışmalarında hem glenohumeral bağ kompleksini anatomisini detaylı olarak tanımladılar hem de glenohumeral bağ kompleksinin kronik omuz instabilitesinde rolü olduğu hipotezini ortaya attılar. ${ }^{[1]} 1906$ yılında Perthes, 1923 yılında ise Bankart bu çalışmayı destekler mahiyette olan çalışmalarını yayımladı. ${ }^{[2]}$ Bankart dört hastada, deltopektoral ve transsubskapular yaklaşımı kullanarak ekleme girdiği bu tekniğinde ipek dikişler kullanarak labrumu glenoide tespit etti ve uzun dönemde rekürrens bildirmedi. ${ }^{[5]}$

Vittorio Putti ve Harry Platt, birbirlerinden bağımSIz bir şekilde 1923-25 yılları arasında kendi tekniklerini tanımladılar. Bu teknikte subskapularis tendonunu lateral insersiyosundan yaklaşık $2,5 \mathrm{~cm}$ uzaklıktan kesilerek oluşturulan miyokapsüler flep glenoid halkaya veya labruma sütüre ediliyordu (Şekil 2). ${ }^{[6]}$ Bu hastaların uzun dönem takiplerinde posterior çıkıkların görülmesi sebebiyle bu teknik yetersiz kaldı. ${ }^{[2]} 1940$ 'lı yıllarda
Magnuson ve Stack subskapularis tendonunu küçük tüberkülümden ayırıp, bisipital oluğa taşıyarak yaptıkları cerrahi tekniklerini yayınladılar (Şekil 3). ${ }^{[7]}$ Bu teknikte amaç omzun anteriorunda gerginliği artırmaktı ancak teknik, posterior çıkık, eksternal rotasyon kısıtlaması, artrit gibi komplikasyonlar sebebiyle istenilen sonucu vermedi. ${ }^{[2]} 1948$ yılında Gallie, fasya lata grefti kullanarak skapula posteriorundan anteriorda humerus ve korakoid çıkıntıya uzanan bir yumuşak doku rekonstrüksiyonu tekniğini bildirdi. ${ }^{[8]}$

1917 yılında Eden, tibiadan alınacak kortikokansellöz kemik bloğunun skapula boynuna, glenoid anterioruna transferini önerdiği ve bundan sonraki buna benzer tekniklerin öncüsü olacak çalışmasını yayımladı. 1932 yılında İsviçre'li cerrah Hybinette tibial kemik bloğu yerine iliak kanattan alınan trikortikal kemik greftini kullandı. [2] 1954 yılında Latarjet ve Trillat eş zamanlı olarak korakoid çıkıntının horizontal bacağının glenoid anterio-inferioruna tespit ederek omzun anteriora dislokasyonun engelledikleri tekniklerini eş zamanlı olarak yayımlamışlardır. Aralarındaki fark, Latarjet'in subskapularisi ve periosteumu ayırarak işlemi yaparken Trillat'ın ise periosteuma dokunmamasıydı. ${ }^{[2,9,10]}$ Bristow da benzer bir teknik tanımlamış olup bu tekniği 1958 yılında öğrencisi Helfet tarafından yayımlanmıştır. ${ }^{[11]}$ Bristow'un tekniğinde korokoid çıkıntı yine kas bağlantıları üzerindeyken alınarak (Latarjet'e göre daha küçük bir parça) subskapularis split yaklaşımla glenoid anterioruna dik bir şekilde 


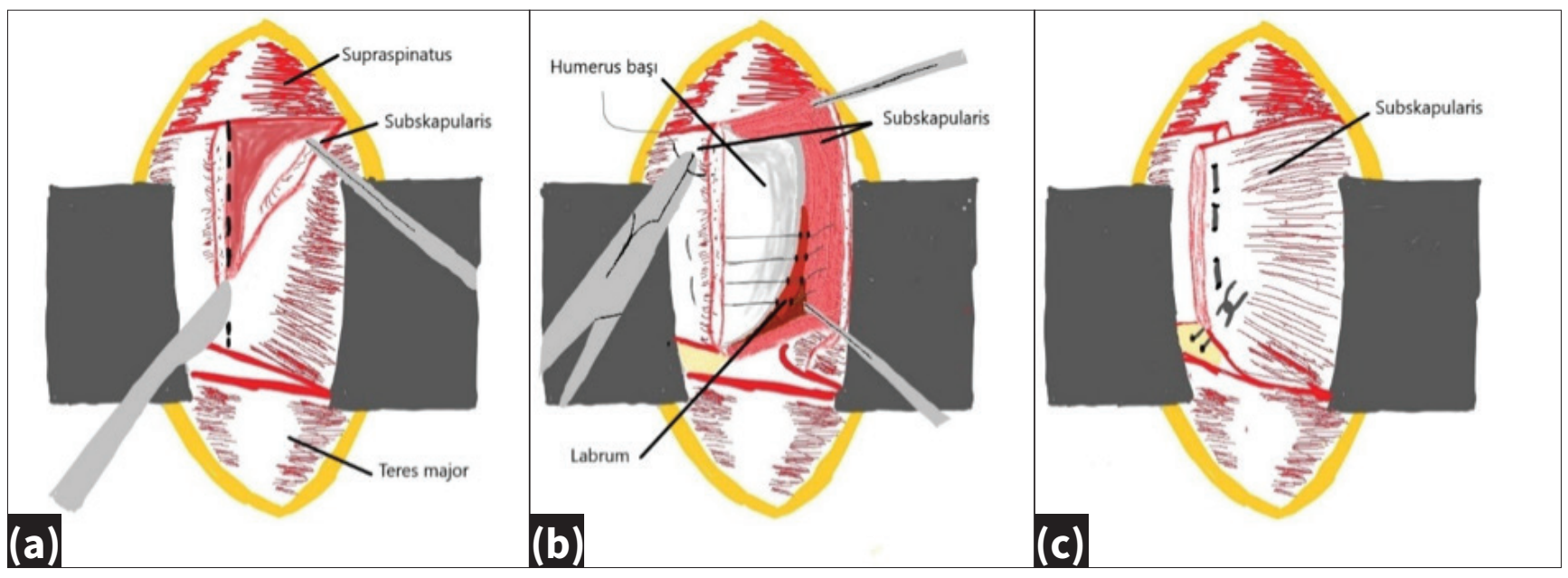

Şekil 2.a-c. Putti-Plat işlemi: Deltopektoral yaklaşımla omuz eklemine girilir. Konjoint tendon ekarte edilir, ardından subskapularis insersiosunun yaklaşık 2,5 cm medialinden kol eksternal rotasyondayken kas vertikal olarak kapsülle beraber kesilir (a). Bu lateral miyokapsüler flap glenoid kenara (rime) ya da labruma ya da medial kapsülün derin yüzüne sütüre edilir (b). Medial miyokapsüler flap de bisipital oluğun medialine sütüre edilir (c).

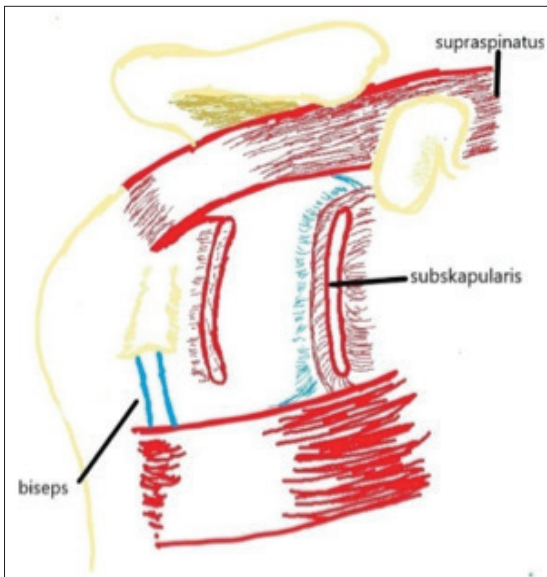

(a)

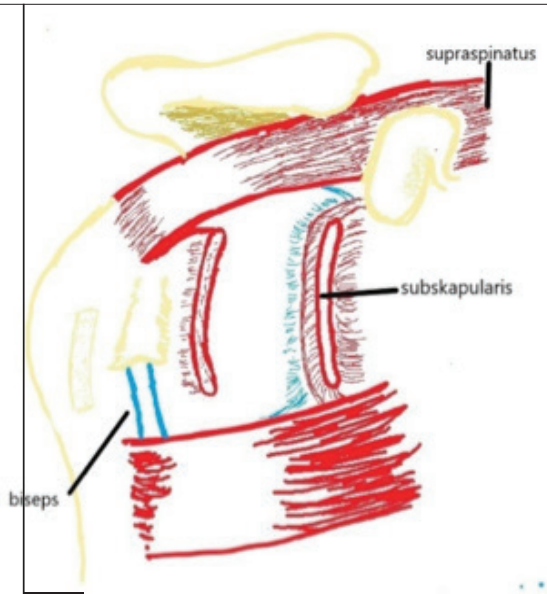

(b)

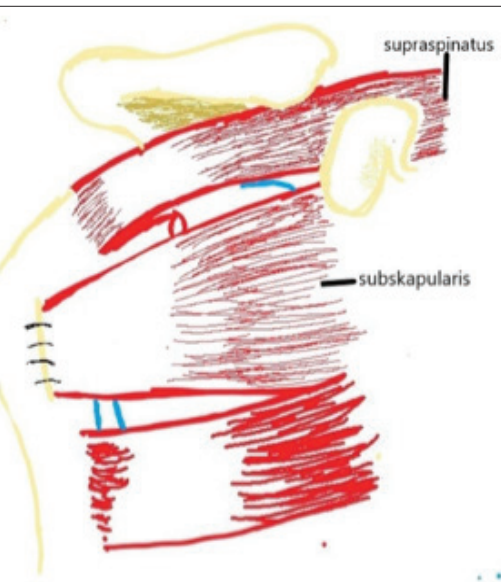

(c)

Şekil 3.a-c. Magnuson-Stack işlemi: Deltopektoral yaklaşımla omuz eklemine girildikten sonra kol dış rotasyondayken subskapularis tendon lateral insersiosundan ya da insersionun biraz medialinden ayrılır (a), kol iç rotasyona alınarak bisipital oluğun lateralinde kemik oluk açılır (b) ve son olarak da subskapularis tendonu yeni insersiosuna dikilir (c).

yerleştiriliyordu (Latarjet kemik kısmı glenoide paralel yerleştiriyordu). ${ }^{[2]}$

Neer ve Foster 1980 yılında ilk kez açık inferior kapsüler kaydırma tekniğini tanımladılar. Bu teknikte lateral tabanlı T şeklinde bir kesi yapılarak inferior kapsüler flep superiora ve laterale taşınıyordu ve superior flep ile destekleniyordu. ${ }^{[12]}$ Sonrasında literatürde değişik kapsüler bindirme teknikleri de bildirildi. ${ }^{[13]}$

ilk rotator kılıf onarımı Avrupa'da 1906 yılında Perthes tarafından, Amerika'da ise 1911 yılında Codman tarafından yapılmıştır. Perthes ilk kez çapa sütürleri kullanarak onarım yaparken Codman ise ipek sütürler ile primer sütüre ederek onarımı yapmıştır. ${ }^{[14,15]} 1944$ yılında Mc Laughlin retrakte lezyonların onarımı için kullandığı tekniğini yayımlamıştır. ${ }^{[16]}$ Debeyre ve Patte 1961 yılında geniş rotator kılıf yırtıklarının tamiri için supraspinatus ilerletme tekniğini tanımlamışlardır. ${ }^{[15]}$

Akromioplasti hâlâ omuz cerrahisinde en sık yapılan işlemlerden biri olup, zaman içerisinde açık tekniklerden artroskopik tekniklere doğru geçiş olduğunu görmekteyiz. 1972 yılında Neer subakromial impingement kavramını tanımladığı çalışmasını yayımladı. Ayrıca bu çalışmasında bu patolojiyi ortadan kaldırmak için geliştirdiği anterior akromioplasti tekniğini de anlatmaktaydı. ${ }^{[17]}$ Yine 1972 yılında Conolly, Hill-Sachs defektine infraspi- 
natus ve kapsülün açık teknikle transferini içeren remplissage (içini doldurma) tekniğini yayımladı. ${ }^{[13]}$

Rotator kılıf yırtıklarının cerrahi tedavisinde tendon transferleri de bir tedavi seçeneği olarak denenmiştir. Illk kez 1982 yılında Cofield subskapularis kasını transfer ettiği tekniğini yayımlamıştır. ${ }^{[18]}$ Gerber ve ark.'nın latissumus dorsi kasını transfer ederek yaptıkları birden fazla çalışma da mevcuttur. ${ }^{[19,20]}$

Bigliani ve ark. 1992 yılında, ilk kez başarısız olan rotator kılıf onarımlarının tekrardan tamir edilmesini önermişlerdir. ${ }^{[21]}$

\section{OMUZ ARTROSKOPISI DÖNEMI}

Illk omuz artroskopisi 1931 yılında Michael Burman tarafından 4 mm'lik rijit endoskop kullanarak kadavra eklemi üzerinde yapılmıştır. ${ }^{[22]} 1970$ 'lerin sonlarına doğru Watanabe ve ark.'nın geliştirdiği artroskopi cihazları ile yapılan ve etkinliği, güvenilirliği giderek artan diz artroskopilerinin sonrasında bu tekniğin omuzda da kullanılabileceği fikri oluşmuştur. ${ }^{[23]}$ Tarihsel süreç incelendiğinde artroskopik instabilite cerrahisinin artroskopik rotator kılıf cerrahisine göre daha önce tanımlandığı ve uygulandığı görülmektedir.

Illk artroskopik stabilizasyon tekniği Lanny Johnson tarafından 1980 yılında tanımlanmıştır. Johnson bu çalışmasında kapsüler zımbalama (stapling) tekniğini kullanarak onarım yapmıştır. ${ }^{[24]}$ Fakat bu teknikte glenoid inferioruna ulaşmak çok zordu ve bu sebeple sonuçlarda yüksek derecede yetmezlik görülmekteydi. ${ }^{[2,13]} 1987$ yılında Morgan ve ark. transglenoidal sütür kullanarak yaptıkları artroskopik Bankart onarımı vakalarını bildirmişlerdir. ${ }^{[25]} 1988$ yılında Caspari transglenoidal sütürlerin ilerletilip, gerginliğinin ayarlanabildiği bir teknik geliştirdi. ${ }^{[26]} \mathrm{Bu}$ iki teknikteki temel sıkıntı düğümlerin infraspinatus fasyası üzerinde sıkılması sebebiyle ilerleyen zamanlarda gelişebilecek gevşemeye bağlı yetmezlik ve supraskapuler sinirin tehdit altında olmasıydı. ${ }^{[13]}$ Wiley benzer bir yaklaşımla metal perçinler kullanarak bir onarım tekniği geliştirdi. Bu teknikte metal perçinler yumuşak doku iyileşmesi sonrası alınıyordu. ${ }^{[27]}$ Warner ve ark. 1995 yılında artroskopik Bankart tamiri için biyobozunur çivileri kullandı. ${ }^{[28]} 1990$ 'ların başında Snyder ve Wolfe ayrı ayrı yaptıkları çalışmalarında çapa sütürleri kullanarak Bankart tamiri yapmışlardır. ${ }^{[29,30]}$ Harryman ve ark. 1994 yılında sadece sütürle labrum tamiri tekniklerini yayımlamışlardır. ${ }^{[31]} 2001$ yılında Thal ve ark. düğümsüz çapa sütürleri Bankart tamirinde kulland.. ${ }^{[32]}$
1988 yılında Snyder ilk kez superior labral anterior-posterior (SLAP) lezyonlarını tanımladı ve artroskopik tedavisini bildirdi. ${ }^{[15]}$

Illk artroskopik subakromial debridman, 1985 yılında Ellman ve ark. tarafından yapılmıştır. ${ }^{[33]}$ Günümüzde birçok rotator kılıf artroskopisi ameliyatının standart bir basamağı haline gelmiştir. 1990 yılında Levy ve ark. 25 hasta üzerinde yaptıkları artroskopi yardımlı mini açık rotator kılıf onarımı sonuçlarını yayımlamışlardır. ${ }^{[34]}$ Snyder ve ark. 1993 yılında çapa sütürleri kullanarak rotator kılıf onarımı yapmışlardır. ${ }^{[15]}$ Burkhart ve ark. 2003 yılında çift sıra rotator kılıf tekniğini yayımlamışlardır. ${ }^{[35]}$ Akabinde Park ve ark. 2007 yılında transosseöz ekivalan tekniğini tanımlamışlardır. ${ }^{[36]}$

Nourussiat ve ark. 2006 yılında kadavra üzerinde yaptıkları artroskopi destekli mini açık Bristow-Latarjet tekniğini yayımladılar. Tam artroskopik Bristow-Latarjet tekniği ise Fransız cerrahlar Lafosse ve Boileau tarafından popülerize edildi. ${ }^{[37,38]}$

Wolf ve ark. 2007 yılında artroskopik Bankart tamirine ek olarak artroskopik posterior kapsülodez ve infraspinatus tenodezini içeren arthroscopic remplissage (içini doldurma) tekniklerini bildirdiler. ${ }^{[39]}$

2007 yılında Mihata ve ark. fasya lata grefti kullanarak artroskopik superior kapsül rekonstrüksiyonu (ASKR) yaptılar. ${ }^{[40]} 2003$ yılında aselüler dermal matriks allogreft GraftJacket piyasaya sürüldü ve 2008 yılında Snyder aselüler dermal matriks allogreft kullanarak yaptığı ASKR sonuçlarını yayımladı. ${ }^{[41]}$

Omuzda instabilite ve rotator kılıf cerrahilerinin tarihsel gelişimini özetlediğimiz bu yazıda görüldüğü üzere her iki başlıkta da ilk önce yaralanmanın tanımlanması, sonrasında tedavide palyatif cerrahi, anatomik olmayan açık yumuşak doku rekonstrüksiyonları, anatomik açık tamir cerrahileri ve bunların artroskopik uygulamaya evrilme süreçleri özetlendi. Tarihi süreçte instabilite cerrahisinin rotator kılıf cerrahisinden daha önce ve daha önde uygulandığını görmekteyiz. Omuz cerrahisinin tarihsel süreci Tablo 1'de özetlenmiştir. Zamanla gelişen teknolojik uygulamaların hem işlemleri daha minimal invaziv bir sürece soktuğu hem de daha kolay uygulanabilir bir hâle getirdiği ortadadır. Bu dinamik süreç hem günümüz cerrahisinde tanımlanmamış ya da tanımlansa da göz ardı edilen bazı lezyonlara olası müdahale sürecine hem de bilinen ve hâlâ kullanılan açık yöntemlerden daha minimal invaziv prosedürlere doğru evrilmektedir. 
Tablo 1. Rotator kılıf - omuz instabilite cerrahisi tarihsel gelişim çizelgesi ${ }^{[2,15]}$

\begin{tabular}{|c|c|c|}
\hline 1788 & Alexander Monro & Suprasinatus ve infraspinatus yırtığının ilk tanımı \\
\hline 1819 & Karl August Weinhold & Redükte edilemez çıkıklar için fibröz bantların subkutanöz ayrııması \\
\hline 1822 & Astley Cooper & Posterior omuz çıkığının ilk medikal tanımı \\
\hline 1834 & John Gregory Smith & Kadavrada ilk rotator kılıf yırtığı serileri \\
\hline \multirow[t]{2}{*}{1855} & Jean-François Malgaigne & Humerus başındaki kemik defektinin ilk tasviri \\
\hline & & Çıkıkların rotator kılıf yırtıkları etiyolojisindeki rolü \\
\hline \multirow[t]{2}{*}{1870} & Emil T. Kocher & Distal manipülasyona dayalı omuz redüksiyonu tekniği \\
\hline & Karl Hüter & $\begin{array}{l}\text { Illk rotator kılıfın humerus diafizine onarımı (kronik çıkıkta humeral baş } \\
\text { rezeksiyonu sonrası) }\end{array}$ \\
\hline 1872 & Simon Emmanuel Duplay & Omuz ağrısında eklem sertliğinin ve yapışıklıkların rolü \\
\hline 1873 & Robert Adams & Rotator kılıf yırtıkları etiyolojisindeki rolü \\
\hline 1878 & Eduard Albert & Redükte edilemez çıkıklar için skapulohumeral artrodez \\
\hline 1882 & Friedrich Cramer & Redükte edilemez çıkıklar için humerus başı rezeksiyonu \\
\hline 1890 & Auguste Broca, Henri A.C.A. Hartmann & Rekürren çıkıklarda kapsülolabral hasarın rolü (hipotez) \\
\hline 1898 & Wilhelm Müller & İnstabilite cerrahisi sırasında ilk rotator kılıf onarımı \\
\hline 1906 & Georg Clemens von Perthes & Avrupa'daki ilk vaka serisi (üç rotator kılıf onarımı) \\
\hline & & Rekürren çıkıklarda kapsülolabral hasarın rolü (kabul) \\
\hline \multirow[t]{2}{*}{1907} & John Sheldon & Kronik posterior çıkıklarda cerrahi redüksiyon \\
\hline & Charles F. Painter & Subdeltoid bölgede kalsifik depozitlerin tanımı \\
\hline 1911 & Ernest Amory Codman & Amerika Birleşik Devletleri'ndeki ilk vaka serisi (iki rotator kılıf onarımı) \\
\hline 1917 & Rudolf Theis Eden & Anterior skapula boynuna tibial kemik bloğu transferi \\
\hline 1923 & Arthur S. B. Bankart & Rekürren anterior omuz çıkıklarında labrumun glenoide tamiri \\
\hline 1923-25 & Vittorio Putti, Harry Platt & Rekürren anterior çıkıklar için Putti-Platt prosedürü \\
\hline 1931 & Michael Samuel Burman & Kadavrada ilk omuz artroskopisi \\
\hline 1932 & Samuel Hybinette & Anterior skapula boynuna iliak kemik bloğu transferi \\
\hline 1940 & Harold A. Hill, Maurice D. Sachs & Hill-Sachs lezyonunun radyolojik tanımı \\
\hline 1943 & Paul B. Magnuson, James K. Stack & Rekürren anterior çıkıklar için Magnuson-Stack prosedürü \\
\hline 1944 & Harrison L. McLaughlin & Retrakte lezyonlar için tedavi tanımı \\
\hline 1949 & Amond Fried & Rekürren posterior çıkık için posterior kemik blok prosedürü \\
\hline 1952 & Harrison L. McLaughlin & Ters Hill-Sachs lezyonunun tanımı ve subskapularis transferi \\
\hline 1954 & Michel Latarjet, Albert Trillat & Anterior glenoid kenara (rime) korakoid transferi \\
\hline 1957 & Masaki Watanabe & Ilk artroskopi atlası \\
\hline 1961 & Jean Debeyre & Büyük lezyonlar için supraspinatus kasının lateral ilerletilmesi \\
\hline 1965 & Frederick R. Thompson & Çok yönlü instabilite vakalarının ilk tanımı \\
\hline 1967 & Dan J. Scott & Rekürren posterior çıkıklar için posterior glenoid osteotomisi \\
\hline
\end{tabular}


Tablo 1. Rotator kılıf - omuz instabilite cerrahisi tarihsel gelişim çizelgesi ${ }^{[2,15]}$ (devamı)

\begin{tabular}{|c|c|c|}
\hline \multirow[t]{2}{*}{1972} & Charles S. Neer & Anterior akromioplasti \\
\hline & John F. Connolly & Açık Hill-Sachs remplissage \\
\hline 1974 & Masaki Watanabe & Uluslararası Artroskopi Derneği'nin kurulması \\
\hline \multirow[t]{2}{*}{1978} & Masaki Watanabe & Omuz artroskopisi için posterior portalin tanımı \\
\hline & Julius S. Neviaser & Rotator kılıf augmentasyonu için ilk otolog skaffold tanımı \\
\hline 1979 & V. Conti & Omuz artroskopisi için anterior portal tanımı \\
\hline \multirow[t]{3}{*}{1980} & Lanny L. Johnson & Kapsüler zımbalama (stapling) ile artroskopik omuz stabilizasyonu \\
\hline & Charles S. Neer, Craig R. Foster & İstemsiz/çok yönlü instabilite için inferior kapsüler kaydırma \\
\hline & Didier Patte & Omuz mikroinstabilite vakalarının ilk tanımı \\
\hline 1983 & Robert Cofield & Subskapularis transferi \\
\hline 1984 & Rolf Ideberg & Eklem içi glenoid kırıkları sınıflaması \\
\hline 1985 & James Andrews & Parsiyel supraspinatus yırtıkları için artroskopik debridman \\
\hline 1986 & Jiro Ozaki & Rotator kılıf augmentasyonu için ilk yapay skaffold tanımı \\
\hline \multirow[t]{5}{*}{1987} & Craig D. Morgan & Anterior omuz çıkıkları için artroskopik labral tekrar tespit \\
\hline & Richard B. Caspari & Rekürren anterior çıkıklar için kapsüloligamentöz germe \\
\hline & Carter R. Rowe & Açık rotator aralık kapaması \\
\hline & Harvard Ellman & ilk büyük artroskopik subakromial dekompresyon serisi (50 vaka) \\
\hline & Paul Grammont & Ters omuz protezi \\
\hline 1988 & Christian Gerber & Masif yırtıklar için latissimus dorsi transferi \\
\hline \multirow[t]{2}{*}{1989} & Eugene M. Wolf & Omuz artroskopisi anterior portallerinin detaylı tanımlanması \\
\hline & Steven C. Thomas, Frederik A. Matsen & Omuz instabilite sınıflaması-TUBS/AMBRI sistemi \\
\hline \multirow[t]{2}{*}{1990} & Stephen J. Snyder & SLAP lezyonunun tanımı \\
\hline & Howard J. Levy & Artroskopi yardımlı rotator kılıf onarımı \\
\hline $1990-91$ & Stephen J. Snyder, Eugene M. Wolf & Artroskopik Bankart onarımı için çapa sütürlerin kullanımı \\
\hline \multirow[t]{2}{*}{1992} & Thomas J. Neviaser & ALPSA lezyonunun tanımı \\
\hline & Louis U. Bigliani & Başarısız rotator kılıf onarımının cerrahi tedavisi \\
\hline \multirow[t]{2}{*}{1993} & Richard Duncan & Çok yönlü instabilite için artroskopik inferior kapsüler kaydırma \\
\hline & Stephen J. Snyder & Ilk artroskopik rotator kılıf onarımı \\
\hline \multirow[t]{3}{*}{1994} & Stephen S. Burkhart & Masif rotator kılıf yırtıkları için parsiyel onarım \\
\hline & Douglas T. Harryman & Basit sütür ile labral onarım \\
\hline & Mark M. Williams, Stephen J. Snyder & Buford kompleksi ve sublabral foramenin tanımı \\
\hline 1995 & Eugene M. Wolf & HAGL lezyonunun tanımlanması \\
\hline $1995-97$ & Larry D. Field, Stephen H. Treacy & Artroskopik rotator interval kapatılması \\
\hline 1996 & Stephen S. Burkhart & Büyük rotator kılıf yırtıkları için marjin-konverjans tekniği \\
\hline
\end{tabular}


Tablo 1. Rotator kılıf - omuz instabilite cerrahisi tarihsel gelişim çizelgesi[2,15] $($ devamı)

\begin{tabular}{|c|c|c|}
\hline 1998 & Scott D. Mair & Posterior ağrılı omuzlarda ters Bankart lezyonu \\
\hline 1999 & Joseph C. Tauro & Retrakte rotator kılıf yırtıkları için artroskopik interval kaydırma \\
\hline \multirow[t]{2}{*}{2000} & Eugene M. Wolf & GAGL lezyonunun tanımlanması \\
\hline & Stephen S. Burkhart & Hill-Sachs angajman konsepti \\
\hline \multirow[t]{2}{*}{2002} & Seung-Ho Kim & Artroskopik revizyon Bankart onarımı \\
\hline & Christian Gerber & Omuz instabilite sınıflaması- anatomik+fonksiyonel özellikler \\
\hline \multirow[t]{2}{*}{2003} & Seung-Ho Kim & Artroskopik posterior labral onarım ve kapsüler kaydırma \\
\hline & Ian K. Lo & Çift sıra artroskopik rotator kılıf tamiri \\
\hline 2004 & Seung-Ho Kim & Kim lezyonunun tanımlanması \\
\hline 2004-08 & Eugene M. Wolf, Robert J. Purchase & Artroskopik Hill-Sachs remplissage \\
\hline \multirow[t]{3}{*}{2006} & Geoffroy Nourissat & Mini-açık Bristow-Latarjet prosedürü \\
\hline & Maxwell C. Park & Transosseöz-ekivalan rotator kılıf onarımı \\
\hline & Nicolas Matis, Nikola Cikak & Artroskopik transosseöz onarım için aletler (ilk yayın girişimleri) \\
\hline \multirow[t]{4}{*}{2007} & Laurent Lafosse & Artroskopik Latarjet prosedürü \\
\hline & Nobuyuki Yamamoto, Eiji Itoi & Hill-Sachs izleme konsepti \\
\hline & Alessandro Castagna & Omuz mikroinstabilitesi için artroskopik müdahale \\
\hline & Enrico Gervasi & Artroskopik latissimus dorsi transferi \\
\hline \multirow[t]{2}{*}{2008} & Pietro Randelli & $\begin{array}{l}\text { Artroskopik rotator kılıf onarımında trombositten zengin plazma kullanımı: } \\
\text { ilk pilot çalışma }\end{array}$ \\
\hline & Ettore Taverna, Markus Scheibel & Artroskopik kemik greft prosedürü (Eden prosedürü) \\
\hline 2009 & Eugenio Savarese & Subakromiyal biyobozunur spacer \\
\hline 2012 & João L. Ellera Gomes & Otolog kök hücre takviyeli rotator kılıf onarımı \\
\hline 2013 & $\begin{array}{l}\text { Chih-Chien Tsai, Pietro Randelli, Hajime } \\
\text { Utsunomiya }\end{array}$ & Omuz dokusundan insan mezenkimal kök hücre izolasyonu \\
\hline 2014 & Noboru Taniguchi & Artroskopik yüzey tutucu onarım \\
\hline
\end{tabular}

TUBS/AMBRI: Traumatic Anterior Shoulder Instability/Atraumatic Multidirectional Bilateral Rehabilitation Inferior capsular shift, SLAP: Superior labrum anterior posterior, ALPSA: Anterior labroligamentous periosteal sleeve avulsion, HAGL: Humeral avulsion of the glenohumeral ligament, GAGL: Glenoid avulsion of the glenohumeral ligament.

\section{KAYNAKLAR}

1. Iqbal S, Jacobs U, Akhtar A, Macfarlane RJ, Waseem M. A history of shoulder surgery. Open Orthop J 2013;7:305-9. Crossref

2. Randelli P, Cucchi D, Butt U. History of shoulder instability surgery. Knee Surg Sports Traumatol Arthrosc 2016;24(2):30529. Crossref

3. Sarban S, Aksoy S, Uzel I, Isikan UE, Atik S. Orthopaedic techniques of Sabuncuoglu in the $15^{\text {th }}$ century Ottoman period. Clin Orthop Relat Res 2005;439:253-9. Crossref
4. Uzel I. Cerrahiyyetü’l Haniyye. Türk Tarih Kurumu Press: Ankara,2020.

5. Bankart AS, Cantab MC. Recurrent or habitual dislocation of the shoulder-joint. 1923. Clin Orthop Relat Res 1993(291):3-6. Crossref

6. Osmond Clarke H. Habitual dislocation of the shoulder; the Putti-Platt operation. J Bone Joint Surg Br 1948;30B(1):19-25. Crossref

7. Magnuson PB, Stack JK. Recurrent dislocation of the shoulder. 1943. Clin Orthop Relat Res 1991(269):4-8; discussion 2-3. Crossref 
8. Gallie WE, Le Mesurier AB. Recurring dislocation of the shoulder. J Bone Joint Surg Br 1948;30B(1):9-18. Crossref

9. Latarjet M. Technic of coracoid preglenoid arthroereisis in the treatment of recurrent dislocation of the shoulder. Lyon Chir 1958;54(4):604-7.

10. Trillat A. Treatment of recurrent dislocation of the shoulder; technical considerations. Lyon Chir 1954;49(8):986-93.

11. Helfet AJ. Coracoid transplantation for recurring dislocation of the shoulder. J Bone Joint Surg Br 1958;40-B(2):198-202. Crossref

12. Neer CS 2nd, Foster CR. Inferior capsular shift for involuntary inferior and multidirectional instability of the shoulder. A preliminary report. J Bone Joint Surg Am 1980;62(6):897-908. Crossref

13. Levy DM, Cole BJ, Bach BR Jr. History of surgical intervention of anterior shoulder instability. J Shoulder Elbow Surg 2016;25(6):e139-50. Crossref

14. Codman EA. Complete rupture of the supraspinatus tendon. Operative treatment with report of two successful cases. 1911. J Shoulder Elbow Surg 2011;20(3):347-9. Crossref

15. Randelli P, Cucchi D, Ragone V, de Girolamo L, Cabitza P, Randelli M. History of rotator cuff surgery. Knee Surg Sports Traumatol Arthrosc 2015;23(2):344-62. Crossref

16. McLaughlin HL. Lesions of the musculotendinous cuff of the shoulder. The exposure and treatment of tears with retraction. 1944. Clin Orthop Relat Res 1994(304):3-9. Crossref

17. Neer CS 2nd. Anterior acromioplasty for the chronic impingement syndrome in the shoulder: a preliminary report. J Bone Joint Surg Am 1972;54(1):41-50. Crossref

18. Cofield RH. Subscapularmuscletransposition for repair of chronic rotator cuff tears. Surg Gynecol Obstet 1982;154(5):667-72.

19. Gerber C, Rahm SA, Catanzaro S, Farshad M, Moor BK. Latissimus dorsi tendon transfer for treatment of irreparable posterosuperior rotator cuff tears: long-term results at a minimum follow-up of ten years. J Bone Joint Surg Am 2013;95(21):1920-6. Crossref

20. Gerber C, Vinh TS, Hertel R, Hess CW. Latissimus dorsi transfer for the treatment of massive tears of the rotator cuff. A preliminary report. Clin Orthop Relat Res 1988(232):51-61. Crossref

21. Bigliani LU, Cordasco FA, Mcllveen SJ, Musso ES. Operative treatment of failed repairs of the rotator cuff. J Bone Joint Surg Am 1992;74(10):1505-15. Crossref

22. Burman MS. Arthroscopy or the direct visualization of joints: an experimental cadaver study. 1931. Clin Orthop Relat Res 2001(390):5-9. Crossref

23. Desai SS. History and evolution of shoulder arthroscopy. J Arthrosc Surg Sports Med 2020;1(1):11-5. Crossref

24. Johnson LL. Arthroscopy of the shoulder. Orthop Clin North Am 1980;11(2):197-204. Crossref

25. Morgan CD, Bodenstab AB. Arthroscopic Bankart suture repair: technique and early results. Arthroscopy 1987;3(2):111-22. crossref
26. Mclntyre LF, Caspari RB. The rationale and technique for arthroscopic reconstruction of anterior shoulder instability using multiple sutures. Orthop Clin North Am 1993;24(1):55-8. Crossref

27. Wiley AM. Arthroscopy for shoulder instability and a technique for arthroscopic repair. Arthroscopy 1988;4(1):25-30. Crossref

28. Warner JJ, Miller MD, Marks P. Arthroscopic Bankart repair with the Suretac device. Part II: Experimental observations. Arthroscopy 1995;11(1):14-20. Crossref

29. Snyder SJ, Karzel RP, Del Pizzo W, Ferkel RD, Friedman MJ. SLAP lesions of the shoulder. Arthroscopy 1990;6(4):274-9. Crossref

30. Wolf EM, Siparsky PN. Glenoid avulsion of the glenohumeral ligaments as a cause of recurrent anterior shoulder instability. Arthroscopy 2010;26(9):1263-7. Crossref

31. Harryman DT 2nd, Ballmer FP, Harris SL, Sidles JA. Arthroscopic labral repair to the glenoid rim. Arthroscopy 1994;10(1):20-30. Crossref

32. Thal R. A Knotless suture anchor: technique for use in arthroscopic Bankart repair. Arthroscopy 2001;17(2):213-8. Crossref

33. Ellman $\mathrm{H}$. Arthroscopic subacromial decompression: analysis of one- to three-year results. Arthroscopy 1987;3(3):173-81. Crossref

34. Levy HJ, Uribe JW, Delaney LG. Arthroscopic assisted rotator cuff repair: preliminary results. Arthroscopy 1990;6(1):55-60. Crossref

35. Lo IK, Burkhart SS. Double-row arthroscopic rotator cuff repair: re-establishing the footprint of the rotator cuff. Arthroscopy 2003;19(9):1035-42. Crossref

36. Park MC, ElAttrache NS, Tibone JE, Ahmad CS, Jun BJ, Lee TQ. Part I: Footprint contact characteristics for a transosseous-equivalent rotator cuff repair technique compared with a double-row repair technique. J Shoulder Elbow Surg 2007;16(4):461-8. Crossref

37. Boileau P, Bicknell RT, El Fegoun AB, Chuinard C. Arthroscopic Bristow procedure for anterior instability in shoulders with a stretched or deficient capsule: the "belt-and-suspenders" operative technique and preliminary results. Arthroscopy 2007;23(6):593-601. Crossref

38. Lafosse L, Lejeune E, Bouchard A, Kakuda C, Gobezie R, Kochhar T. The arthroscopic Latarjet procedure for the treatment of anterior shoulder instability. Arthroscopy 2007;23(11):1242 e1-5. Crossref

39. Buza JA 3rd, lyengar JJ, Anakwenze OA, Ahmad CS, Levine WN. Arthroscopic Hill-Sachs remplissage: a systematic review. J Bone Joint Surg Am 2014;96(7):549-55. Crossref

40. Mihata T, McGarry MH, Pirolo JM, Kinoshita M, Lee TQ. Superior capsule reconstruction to restore superior stability in irreparable rotator cuff tears: a biomechanical cadaveric study. Am J Sports Med 2012;40(10):2248-55. Crossref

41. Bond JL, Dopirak RM, Higgins J, Burns J, Snyder SJ. Arthroscopic replacement of massive, irreparable rotator cuff tears using a GraftJacket allograft: technique and preliminary results. Arthroscopy 2008;24(4):403-9 e1. Crossref 